Kompass

Neumología

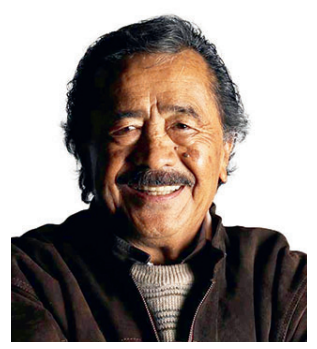

Favio Gerardo Rico-Méndez

Editor Jefe

\title{
Enfermedades pulmonares obstructivas: Medicina personalizada para asma y EPOC
}

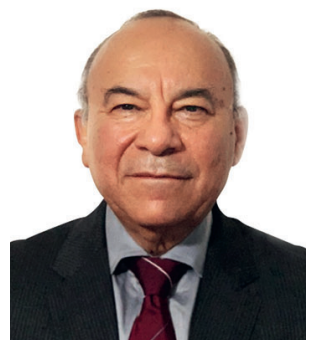

Francisco Espinosa-Larrañaga Coeditor
Hace 62 años se llevó a cabo el primer Simposium llevado a cabo con auspicio de la Fundación CIBA, en dónde se propuso la integración de la bronquitis crónica y el enfisema pulmonar como una misma entidad para su estudio y abordaje clínico con resultados parciales por la reticencia de muchos investigadores para aceptar un nuevo paradigma. Debió de pasar el tiempo, y el que otras instituciones dedicadas a las enfermedades respiratorias decidieran considerar esta visión, como lo hicieron la American Thoracic Society, qué conjuntó voluntades y con ello logró integrar el concepto de lo que hoy conocemos como Enfermedad Pulmonar Obstructiva Crónica (chronic obstructive pulmonary disease, COPD siglas en inglés) y con la colaboración de Consejo de Investigación Médica de Londres (Medical Research Council) se publicó un documento en la revista «The Lancet» en el año de 1965 con el titulo de «Definition and classification of chronic bronchitis for clinical and epidemiological purposes. A report to the Medical Research Council by their committee on the aetiology of chronic bronchitis».

Al momento actual se han modificado conceptos, términos, formas de diagnóstico y tratamiento con el desarrollo de investigaciones y aportaciones al conocimiento que han sido enunciados en la Iniciativa GOLD (IG). La IG conjunta a expertos del mundo en este tema y quienes se han encargado del desarrollo de guías de práctica clínica, las cuales, hasta el momento son las más utilizadas a nivel mundial para la atención de los pacientes que padecen estos padecimientos. No todo está dicho sobre la enfermedad pulmonar obstructiva crónica (EPOC), aún queda mucho por realizar e investigar, dado que las cifras de morbi-mortalidad han sufrido un incremento de acuerdo al estudio de la carga de morbilidad, financiado por la Fundación Bill \& Melinda Gates, la prevalencia del EPOC en el año de 2016 fue de 251 millones de casos y se estima que un año antes (2015) murieron por esta causa, cerca de 3.17 millones de personas en el mundo lo que representa el $5 \%$ de todas las muertes registradas en ese año. Lo más trascendente es que mas del 90\% de los fallecimientos se produjeron en aquellos países de medianos y bajos ingresos en donde el tabaquismo, la contaminación intra y extramuros y la mala higiene industrial son los principales elementos causales de la enfermedad.

Es de esperar que mientras no se tomen medidas adecuadas como aumentar la sensibilización acerca de la epidemia mundial de enfermedades no trasmisibles, buscar la creación e integración de ambientes saludables, abatir el tabaquismo y las diferentes fuentes contaminantes, la enfermedad seguirá en aumento, así como el grado de discapacidad que produce y lo lleva a ocupar la quinta causa mundial y la 13 ava 
en Latinoamérica con un costo de atención que se eleva a varios billones de dolares. También otras enfermedades tienden a incrementarse en forma considerable como el asma, los trastornos del sueño y co-morbilidades relacionadas. Lo anterior está dando como resultado una saturación de los servicios de salud y un aumento en número de ingresos hospitalarios, así como de incapacidad parcial o total para enfrentar el problema, lo que obliga a una nueva visión menos curativa y más preventiva a fin de que los gastos no lleguen a ser considerados catastróficos, a tal grado que colapsen en un futuro los sistemas de salud del mundo.
Cada paciente debe ser atendido de manera personalizada y exhaustiva, a fin de que reciban el tratamiento acorde a su enfermedad, mecanismo de producción de la misma y las diversas medidas preventivas que permita su reintegración a la vida social, familiar y laboral.

En el número en sus manos, se lleva a cabo un análisis desde el punto de vista de la medicina personalizada sobre entidades que estan tomando un auge mundial y causan un gran impacto social, económico y para los sistemas de salud. Cada paciente debe ser atendido de manera personalizada y

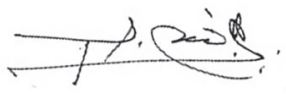

Dr. Favio Gerardo Rico-Méndez Editor Jefe exhaustiva, a fin de que reciban el tratamiento acorde a su enfermedad, mecanismo de producción de la misma y las diversas medidas preventivas que permita su reintegración a la vida social, familiar y laboral.

Deseamos que esta edicion sea para el lector una herramienta útil que le permita vislumbrar nuevos caminos y armas terapéuticas para que su paciente logre una respuesta mas adecuada a su problema de salud específico y apoye las medidas preventivas a fin de abatir tan serias, problemáticas y costosas enfermedades.

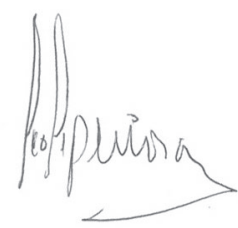

Dr. Francisco Espinosa-Larrañaga Coeditor 